\title{
Insomnia in Sweden: A Population-Based Survey
}

\author{
Lena Mallon, ${ }^{1}$ Jan-Erik Broman, ${ }^{1}$ Torbjörn Åkerstedt, ${ }^{2}$ and Jerker Hetta ${ }^{3}$ \\ ${ }^{1}$ Department of Neuroscience, Psychiatry, Uppsala University, SE-75185 Uppsala, Sweden \\ ${ }^{2}$ Stress Research Institute, Stockholm University, SE-10691 Stockholm, Sweden \\ ${ }^{3}$ Department of Clinical Neuroscience, Psychiatry, Karolinska Institutet, SE-14186 Stockholm, Sweden
}

Correspondence should be addressed to Lena Mallon; lena.mallon@ltdalarna.se

Received 1 March 2014; Revised 28 April 2014; Accepted 1 May 2014; Published 12 May 2014

Academic Editor: Michel M. Billiard

Copyright (C) 2014 Lena Mallon et al. This is an open access article distributed under the Creative Commons Attribution License, which permits unrestricted use, distribution, and reproduction in any medium, provided the original work is properly cited.

\begin{abstract}
Aims. Estimate the prevalence of insomnia and examine effects of sex, age, health problems, sleep duration, need for treatment, and usage of sleep medication. Methods. A sample of 1,550 subjects aged 18-84 years was selected for a telephone interview. The interview was completed by 1,128 subjects $(72.8 \%)$. Results. $24.6 \%$ reported insomnia symptoms. Insomnia disorder, that is, insomnia symptoms and daytime consequences, was reported by $10.5 \%$. The prevalence was similar among all age groups, with the exception of women aged 40-49 years who demonstrated a significantly higher prevalence, $21.6 \%$. Having at least one physical or psychiatric disorder was reported by $82.8 \%$ of subjects with insomnia disorder. Mean sleep duration for subjects with insomnia disorder was 5.77 hours on weeknights and 7.03 hours on days off/weekends. The corresponding figures for subjects without insomnia disorder were 7.04 hours and 7.86 hours, respectively. Among those with insomnia disorder $62.5 \%$ expressed a need for treatment, and $20.0 \%$ used prescribed sleep medication regularly. Conclusions. Insomnia disorder is highly prevalent in the population. There are significant associations between insomnia disorder and physical and psychiatric disorders. A majority of subjects with insomnia disorder expressed a need for treatment, indicating a public health problem.
\end{abstract}

\section{Introduction}

Several epidemiological studies have been conducted in order to estimate the prevalence of insomnia in the general population. The reported prevalence rates vary considerably, and differences in how insomnia is defined contribute to this variation.

Also, some variability may be explained by differences in how information is obtained, that is, questionnaire or interview surveys.

Some studies report insomnia symptoms, that is, difficulties initiating and/or maintaining sleep, without restrictive criteria, while others include duration, frequency, or severity criteria. Studies without restrictive criteria produce prevalence estimates from $25 \%$ to $48 \%$ [1], while studies using some form of duration, frequency, or severity gradations report prevalence rates from $9 \%$ to $34 \%[1,2]$. The diagnosis of insomnia disorder according to classification systems, such as the DSM-IV [3], ICD-10 [4], or ICSD-2 [5] also requires daytime impairment, and with this definition the prevalence rates of insomnia decrease to about $6 \%$ to $12 \%[6,7]$. A consistent finding is that women are more likely to have insomnia [8]. Almost all studies report an increase in insomnia symptoms with age $[9,10]$, while there are mixed results concerning insomnia disorder $[6,7]$.

Subjects with insomnia generally report shorter sleep duration compared to normal sleepers [6], and high levels of comorbidity between insomnia and medical disorders have been demonstrated in epidemiological surveys [11, 12]. Among physical disorders heart disease, hypertension, breathing problems, urinary problems, and pain conditions are more common in subjects with insomnia. Moreover, a survey reported that about half of subjects with insomnia also met criteria for a psychiatric disorder [13]. Although effective behavioural and pharmacological therapies exist for insomnia, many insomnia sufferers do not seek help [14] or use sleep medication $[6,14]$.

It is important to have accurate estimates of the sex- and age-related prevalence of insomnia and its correlates in the general population in order to understand the public health 
effect of the disorder. Given the differences in the definition of insomnia in epidemiological research it is difficult to draw conclusions about the true prevalence of the disorder.

This study aims to evaluate the prevalence of insomnia and to identify factors associated with insomnia in the general adult population in Sweden. We used a definition of insomnia disorder that includes insomnia symptoms and detrimental effects on daytime functioning, and our definition of insomnia disorder is thereby close to the DSM-IV insomnia disorder diagnosis [3].

\section{Methods}

2.1. Procedure. The study was initiated by the Swedish Council on Health Technology Assessment (SBU). Data were collected by a telephone interview commissioned to the Central Bureau of Statistics (SCB), a governmental agency, in Sweden. Data collection was done with a software package designed specifically for this type of computer-assisted phone survey. After a brief description of the aims of the study and after obtaining verbal consent to proceed with the interview, data were collected.

2.2. Participants. A sample of 1,550 subjects living in Sweden, 18-84 years of age, representative for the population and proportionally stratified for age and sex was selected for a telephone interview. The interview was completed by 1128 subjects $(72.8 \%)$. The characteristics of the sample are shown in Table 1 . The sample consisted of $52.1 \%$ women and the mean age was 47.8 years $(\mathrm{SD}=18.0)$.

2.3. Material. The interview consisted of 39 questions covering demographics, work conditions, sleep complaints, daytime impairment due to sleep complaints, sleep duration, physical and psychiatric disorders, need for treatment, and usage of prescriptive sleep medication.

Sleep initiation problems were assessed by asking "How often have you had difficulties falling asleep during the last month?" to be answered on a five-point scale $(1=$ never or less than once a month; $2=$ less than once a week; $3=1-2$ times per week; $4=3-5$ times per week; $5=$ daily or almost daily). Sleep maintenance problems were assessed by asking "How many times do you wake up during the night?" to be answered on a five-point scale $(1=$ never; $2=$ once; $3=$ twice; $4=$ 3-4 times; $5=$ at least 5 times). Insomnia symptoms were defined as sleep initiation problems at least 3 times per week (scores 4 and 5) and/or sleep maintenance problems at least 3 times per night (scores 4 and 5).

Daytime consequences were assessed by asking "Have your sleep complaints interfered with your daily life during the last month?" ( 1 = no interference, $2=$ minor interference; $3=$ moderate interference; $4=$ severe interference; $5=$ very severe interference).

Insomnia disorder was defined as having insomnia symptoms and at least moderate interference with daytime functioning (scores 3 to 5 ).

Sleep duration was assessed by asking subjects to estimate sleep duration on weeknights and on days off/weekends.
TABLE 1: Characteristics of the sample $(N=1128)$.

\begin{tabular}{|c|c|c|c|c|}
\hline & \multicolumn{2}{|l|}{ Men } & \multicolumn{2}{|c|}{ Women } \\
\hline & $N$ & $\%$ & $N$ & $\%$ \\
\hline \multicolumn{5}{|l|}{ Age groups (years) } \\
\hline $18-29$ & 117 & 21.7 & 106 & 18.0 \\
\hline $30-39$ & 102 & 18.9 & 89 & 15.1 \\
\hline $40-49$ & 87 & 16.1 & 111 & 18.9 \\
\hline $50-59$ & 78 & 14.4 & 90 & 15.3 \\
\hline $60-69$ & 95 & 17.6 & 101 & 17.2 \\
\hline $70-84$ & 61 & 11.3 & 91 & 15.5 \\
\hline \multicolumn{5}{|l|}{$\begin{array}{l}\text { Physical and psychiatric } \\
\text { disorders }\end{array}$} \\
\hline Hypertension & 68 & 12.6 & 107 & 18.2 \\
\hline Asthma & 68 & 12.6 & 73 & 12.4 \\
\hline Heart disease & 37 & 6.9 & 39 & 6.6 \\
\hline Diabetes & 35 & 6.5 & 27 & 4.6 \\
\hline Gastrointestinal disorder & 86 & 15.9 & 134 & 22.8 \\
\hline Urogenital disorder & 37 & 6.9 & 36 & 6.1 \\
\hline Cancer & 17 & 3.1 & 14 & 2.4 \\
\hline Joint pain & 98 & 18.1 & 172 & 29.3 \\
\hline Fibromyalgia & 2 & 0.4 & 20 & 3.4 \\
\hline Other physical disorders & 45 & 8.3 & 90 & 15.3 \\
\hline Psychiatric disorder & 26 & 4.8 & 41 & 7.0 \\
\hline Burnout & 19 & 3.5 & 35 & 6.0 \\
\hline Depression & 42 & 7.8 & 70 & 11.9 \\
\hline \multicolumn{5}{|l|}{ Sleep duration (hours) } \\
\hline Weeknights (mean \pm SD) & $6.87 \pm 1.12$ & & $6.93 \pm 1.24$ & \\
\hline $\begin{array}{l}\text { Weekends/days off } \\
(\text { mean } \pm \text { SD })\end{array}$ & $7.85 \pm 1.51$ & & $7.70 \pm 1.55$ & \\
\hline
\end{tabular}

The answer was expressed as a continuous variable. Physical and psychiatric disorders were ascertained by asking (in a yes/no question) if respondents had hypertension, asthma, heart disease, diabetes, gastrointestinal disorder, urogenital disorder, cancer, joint pain, fibromyalgia, psychiatric disorder, burnout, depression, or any other disorder.

Subjects were asked (in a yes/no question) "Do you think you need treatment for your sleep problems?" Usage of prescriptive sleep medication was ascertained by the question "How often during the last month have you used prescriptive sleep medication?" $(1=$ never or less than once per month, $2=$ less than once per week, $3=1-2$ times per week, $4=3-5$ times per week, and $5=$ daily or almost daily). Usage at least 3 times per week (scores 4 and 5) was considered regular usage. Unfortunately we do not have any information about the nature of sleep medication. The type of sleep medication asked about was "on prescription," that is, not sold over the counter. In Sweden the dominating drugs in this class are zopiclone, zolpidem, and propiomazine.

2.4. Data Analyses. All analyses were carried out using IBM SPSS Statistics, version 20.0. Standard methods were used to calculate mean values and standard deviations (SDs). 
TABLE 2: Prevalence (\%) and odds ratios (95\% confidence interval) of insomnia symptoms and insomnia disorder by age groups in men $(n=537)$ and women $(n=587)$.

\begin{tabular}{ccccc}
\hline & \multicolumn{2}{c}{ Insomnia symptoms } & \multicolumn{2}{c}{ Insomnia disorder } \\
& Men & Women & & Men \\
Age groups (yrs) & & & & \\
$18-29$ & $16.2[10.6-24.0]$ & $23.6[16.5-32.5]$ & $6.8[3.5-12.9]$ & $9.4[5.2-16.5]$ \\
$30-39$ & $17.6[11.5-26.2]$ & $32.6[23.7-42.9]$ & $6.9[3.5-12.9]$ & $13.5[7.9-22.1]$ \\
$40-49$ & $18.4[11.6-27.8]$ & $26.1[18.8-35.0]$ & $11.5[6.4-19.9]$ & $21.6[15.0-30.2]$ \\
$50-59$ & $15.4[9.0-25.0]$ & $27.8[19.6-37.8]$ & $5.1[2.0-12.5]$ & $13.3[7.8-21.9]$ \\
$60-69$ & $21.1[14.1-30.3]$ & $30.7[22.5-40.3]$ & $4.2[1.6-10.3]$ & $12.9[7.7-20.8]$ \\
$70-84$ & $31.1[20.9-43.6]$ & $36.3[27.1-46.5]$ & $8.2[3.5-17.8]$ & $9.9[5.3-17.7]$ \\
\hline Total & $19.4[16.2-22.9]$ & $29.3[25.8-33.1]$ & $7.1[5.2-9.6]$ & $13.6[11.1-16.6]$ \\
\hline
\end{tabular}

TABLE 3: Sleep duration on week nights and on weekends/days off in subjects with insomnia disorder $(n=118)$ and in subjects without insomnia disorder $(n=1005)$.

\begin{tabular}{lcccc}
\hline & \multicolumn{2}{c}{ Sleep duration on week nights (hrs) } & \multicolumn{3}{c}{ Sleep duration on weekends/days off (hrs) } \\
& Mean & SD & Mean & SD \\
\hline Subjects with insomnia disorder & 5.77 & 1.64 & $7.04^{* * *}$ & 2.41 \\
Subjects without insomnia disorder & 7.03 & 1.05 & $7.86^{* * *}$ & 1.37 \\
\hline
\end{tabular}

SD: standard deviation: ${ }^{* * *} P<.001$.

When the comparison involved continuous variables the Mann-Whitney $U$ test was used, and the chi-square test was used for differences between proportions.

Ninety-five percent confidence intervals were calculated for prevalence rates and odds ratios. Linear regression models were used to calculate correlations between continuous variables. To identify associations between insomnia disorder and physical and mental disorders age-adjusted and multivariate logistic regression analyses were conducted. Results are presented as odds ratios (OR) with $95 \%$ confidence intervals.

\section{Results}

3.1. Prevalence of Insomnia Symptoms. The prevalence of having difficulties initiating sleep only was $10.7 \%$, and the prevalence of having sleep maintenance problems only was also $10.7 \%$. The prevalence of insomnia symptoms, that is, sleep initiation problems and/or sleep maintenance problems, was $24.6 \%$. Women reported insomnia symptoms more often than men, $29.3 \%$ versus $19.4 \%\left(\chi^{2}=14.9 ; P<.001\right)$. In all age groups insomnia symptoms were more frequent in women (Table 2). Insomnia symptoms were significantly predicted by sex (OR, 1.69; 95\% CI, 1.28-2.23; $P<.001$ ). The highest prevalence of insomnia symptoms was in the oldest age group, 70 to 84 years, in which $31.1 \%$ of men and $36.3 \%$ of women reported insomnia symptoms. With the youngest age group as a reference we found that insomnia symptoms remained comparable between age groups with the exception of men aged 70 to 84 years who had a significant higher prevalence rate (OR, 2.33 95\% CI, 1.12-4.85; $P<.05)$.

3.2. Prevalence of Insomnia Disorder. Of those with insomnia symptoms $42.9 \%$ reported concomitant impairment of daytime functioning and were classified as having insomnia disorder. The prevalence of insomnia disorder was $10.5 \%$, and more women than men reported insomnia disorder, $13.6 \%$ versus $7.1 \%\left(\chi^{2}=12.7 ; P<.001\right)$. In all age groups insomnia disorder was more frequent in women (Table 2). Thus, insomnia disorder was significantly predicted by sex (OR, $2.08 ; 95 \%$ CI, $1.38-3.12 ; P<.001)$. The prevalence of insomnia disorder remained comparable between age groups with the exception of women aged 40 to 49 years who demonstrated a significant higher prevalence rate, $21.6 \%(\mathrm{OR}, 2.65 ; 95 \% \mathrm{CI}$, $1.20-5.85 ; P<.05)$.

3.3. Sleep Duration. Subjects with insomnia disorder reported shorter sleep duration on weeknights compared to subjects without insomnia disorder, $5.77 \pm 1.64$ hours versus $7.03 \pm 1.05$ hours $(t=11.2 ; P<.0001)$. They also reported shorter sleep duration on days off/weekends, $7.04 \pm 2.41$ hours, compared to subjects without insomnia disorder, $7.86 \pm 1.37$ hours $(t=15.9 ; P<.0001)$ (Table 3$)$. Both groups extended their sleep on days off/weekends. On days off/weekends $42.4 \%$ of subjects with insomnia disorder and $43.4 \%$ of subjects without insomnia disorder extended their sleep with at least one hour.

3.4. Comorbid Disorders. Having at least one physical or psychiatric disorder was reported by $82.8 \%$ of subjects with insomnia disorder compared to $54.2 \%$ of subjects without insomnia disorder (OR, 1.78; 95\% CI, 1.58-1.98; $P<.001$ ). In subjects with insomnia disorder there was no sex difference in the prevalence of comorbid disorders (OR, 0.74; 95\% CI, $0.24-2.24$; n.s.), but there was a difference between age groups (OR, 1.72; 95\% CI, 1.20-2.48; $P<.01$ ). The prevalence of comorbid disorders increased with advancing age, and all of the insomniacs aged 60 and above reported at least 
TABLE 4: Prevalence (\%) and odds ratios (95\% confidence interval) of physical and psychiatric disorders in subjects without insomnia disorder $(n=1005)$ and in subjects with insomnia disorder $(n=118)$.

\begin{tabular}{|c|c|c|c|c|}
\hline & $\begin{array}{c}\text { Subjects without } \\
\text { insomnia disorder } \\
\%\end{array}$ & $\begin{array}{c}\text { Subjects with insomnia } \\
\text { disorder } \\
\%\end{array}$ & $\begin{array}{c}\text { Subjects with insomnia; } \\
\text { univariate analyses }{ }^{\mathrm{a}} \\
\text { OR (95\% CI) }\end{array}$ & $\begin{array}{l}\text { Subjects with insomnia; } \\
\text { multivariate analyses } \\
\text { OR }(95 \% \mathrm{CI})\end{array}$ \\
\hline Hypertension & 14.8 & 22.4 & $1.67(1.04-2.67)$ & $0.98(0.53-1.83)$ \\
\hline Asthma & 10.9 & 25.6 & $2.81(1.77-4.44)$ & $1.96(1.11-3.44)$ \\
\hline Heart disease & 5.9 & 14.4 & $2.69(1.51-4.80)$ & $1.13(0.50-2.54)$ \\
\hline Diabetes & 5.1 & 8.5 & $1.75(0.86-3.54)$ & $1.53(0.67-3.51)$ \\
\hline Gastrointestinal disorder & 16.3 & 45.8 & $4.32(2.90-6.44)$ & $2.36(1.46-3.82)$ \\
\hline Urogenital disorder & 5.8 & 12.7 & $2.38(1.30-4.34)$ & $1.72(0.80-3.66)$ \\
\hline Cancer & 2.8 & 2.5 & $0.91(0.27-3.04)$ & $0.30(0.07-1.24)$ \\
\hline Joint pain & 21.3 & 46.6 & $3.22(2.18-4.76)$ & $1.91(1.16-3.15)$ \\
\hline Fibromyalgia & 1.1 & 9.5 & $9.41(3.98-22.23)$ & $5.04(1.88-13.53)$ \\
\hline Other physical disorder & 11.2 & 18.6 & $1.83(1.10-3.02)$ & $1.16(0.63-2.13)$ \\
\hline Psychiatric disorder & 3.7 & 24.1 & $8.32(4.86-14.23)$ & $2.00(0.91-4.37)$ \\
\hline Burnout & 2.5 & 23.7 & $12.17(6.81-21.76)$ & $2.19(0.99-4.85)$ \\
\hline Depression & 6.2 & 40.5 & $10.40(6.64-16.28)$ & $4.91(2.63-9.17)$ \\
\hline
\end{tabular}

OR: odds ratio; CI: confidence interval findings are significant when CIs do not include 1.00 .

${ }^{a}$ Adjusted for age in 5-year strata.

${ }^{\mathrm{b}}$ Multivariate analyses adjusted for age in 5-year strata, all physical and psychiatric disorders.

one physical or psychiatric disorder. All disorders, with the exception of diabetes and cancer, were more common in subjects with insomnia disorder (Table 4). In unadjusted logistic regression analyses significant associations were found between insomnia disorder and several of the disorders. In multivariate logistic regression analysis including all disorders, the association between insomnia disorder and many of the disorders were reduced to a nonsignificant level. Significant associations remained between insomnia disorder and asthma, gastrointestinal disorders, joint pain, fibromyalgia, and depression. The strongest association found was with depression (OR, 4.91; 95\% CI, 2.63-9.17; $P<.001$ ). In subjects with insomnia disorder there was no sex difference (OR, 0.78 ; 95\% CI, 0.36-1.71; n.s.), or difference between age groups (OR, 0.97; 95\% CI, 0.77-1.21; n.s.) in the prevalence of depression.

3.5. Need for Treatment and Usage of Prescriptive Sleep Medication. Among those with insomnia disorder 62.5\% expressed a need for treatment, and $22.0 \%$ used prescriptive sleep medication regularly. There was no age or sex difference in expressed need for treatment or usage of sleep medication. Subjects with insomnia disorder who were depressed used sleep medication more often than insomniacs without depression, $33.3 \%$ versus $17.1 \%$ (OR, 3.00; 95\% CI, 1.22-7.37; $P<.05)$.

\section{Discussion}

The main finding of the present study is that the prevalence of insomnia disorder is $10.5 \%$, and women are 2.08 more likely to report insomnia disorder. Insomnia disorder did not increase with advancing age since being older decreased the probability of daytime impairment due to insomnia symptoms. There was, however, a significant rise in reports of insomnia disorder in women aged $40-49$ years. Subjects with insomnia disorder slept less than 6 hours on weeknights, but $42.4 \%$ were able to extend sleep with more than 1 hour on days off/weekends. There was a strong overlap between insomnia disorder and physical and psychiatric disorders, most often with depression. A majority of subjects with insomnia disorder expressed a need for treatment, indicating that they are troubled and worried about their sleep, while $20.0 \%$ used sleep medication.

Advantages of the present study include the nationally representative sample, the high response rate, the broad age range, the comprehensive definition of insomnia disorder, and the wide range physical and psychiatric disorders included. Data were collected by telephone interview, and telephone interviews assessing DSM-IV psychiatric disorders have been shown to yield results comparable to other strategies [15].

One limitation is that sleep maintenance problems were assessed by the number of awakenings, and an additional question about duration of wake time would have been good. However, we think that 3 or more awakenings will include most individuals with sleep maintenance problems. This categorization has been shown to be related to daytime symptoms [16]. Individuals with lor 2 long awakenings should of course be regarded as having problems, but when we examined sleep duration in this group there were only few subjects with sleep duration less than 6 hours.

The measure of physical and psychiatric health according to number of disorders reported is relatively coarse but common in epidemiologic studies. The reliability of some selfreported diagnoses, for example, diabetes, is good [16], but others have shown poor reliability [17]. In the present study 
occurrence of depression was based on subjects' response to a single question about having depression. Previously the value of single-item depression screening has been established [18]. A limitation is that certain physical and psychiatric disorders, life style factors such as excessive drinking, and other concomitant medication than sleep-promoting medication which we did not deal with in this study could influence the results. Another restraint is that the question about sleep medication usage focused on prescribed medications.

This study demonstrates that subjects with insomnia disorder had a higher prevalence of several physical and psychiatric disorders compared to subjects without insomnia disorder. The cross-sectional nature of the study does not permit us to disentangle cause and effect; we can only demonstrate associations. Insomnia disorder and other health problems are either causally related to each other or other factors may influence this relationship. As such, we cannot say that insomnia disorder is a result of a physical or psychiatric disorder or that insomnia caused or exacerbated the disorder. However, from longitudinal studies we know that insomnia disorder actually plays a role in disorder development [1922].

One aim of the present study was to provide valid estimates of the prevalence of insomnia disorder. Our definition of insomnia disorder is based on insomnia symptoms with frequency criteria, accompanying daytime consequences and a duration criterion of four weeks, and is thereby close to the DSM-IV insomnia disorder diagnosis [3]. The prevalence of insomnia disorder in our study was $10.5 \%$ and is similar to that of other studies $[7,14,23]$.

In this study insomnia disorder was more common in women, but we found no association with advancing age. There was, however, a significant rise in insomnia disorder in women aged 40 to 49 years. Other surveys have also demonstrated a rise in insomnia disorder frequency in middle rather than old age $[12,24]$.

It has been shown that women more easily express emotional distress and somatic symptoms, like sleep complaints, compared to men [25]. It has also been shown that there are gender differences in coping styles [26] and exposure to stressful life events [27]. Menopause has been suggested as an explanation for the discrepancy between men and women in the prevalence of insomnia in mid-life. A recent study showed that postmenopausal women (53-58 yrs) had more nocturnal awakenings compared to premenopausal women (44-48 yrs), but the frequency of difficulty falling asleep, snoring, and use of sleep medication did not differ between the groups [28].

Although the elderly have difficulties initiating and maintaining sleep many do not report daytime impairment. With advancing age sleep becomes more fragmented and "lighter" due to increased percentage of stage one sleep and decreased percentage of slow wave sleep [29]. It has been shown that there is an age-related reduction in sleep duration and depth required to maintain daytime alertness [30] and that reduced night-time sleep quality in older people does not cause increased daytime sleep propensity [31]. These changes appear to be, at least in part, related to an age-related reduction in the homeostatic drive for sleep and a reduced strength of the circadian signal [32]. Furthermore, a study showed that age was not predictive of insomnia when social satisfaction and activity status were controlled [33], suggesting that life style changes and inactivity accompanying old age can contribute to the lack of reports of daytime impairment.

Generally epidemiological surveys only assess sleep duration without comparing sleep on weeknights to sleep on days off/weekends. In agreement with previous studies subjects with insomnia disorder reported shorter sleep duration than subjects without insomnia disorder [6]. Surprisingly, we found that $42.4 \%$ of subjects with insomnia disorder increased their sleep duration with at least one hour on days off/weekends. This may partly be explained by a reduced stress experienced during weekends. Still it casts some doubts about these subjects having true insomnia disorder. This aspect has not been studied extensively earlier but should indeed be investigated in future studies. Our findings run counter to a survey from Spain demonstrating that subjects with insomnia did not to extend their time in bed on weekends [6] but is in agreement with a study from South Korea where subjects with difficulties initiating sleep slept at least three extra hours on days off and weekends [34].

Our study confirms that insomnia disorder rarely occurs alone. It is by far more common as a comorbid condition than as a single sleep problem. We found that $82.8 \%$ of subjects with insomnia disorder reported one or more disorders which is similar to findings from a survey where $86.1 \%$ of subjects with insomnia reported medical health problems [11]. Depression was reported by $40.5 \%$ of subjects with insomnia disorder, and subjects with insomnia disorder were 4.91 times as likely to have depression compared to subjects without insomnia disorder. We found no variation in association between insomnia disorder and depression between age groups, indicating that depression and insomnia disorder are related to each other with similar strength across life. In addition, we found that fibromyalgia, gastrointestinal disorder, and asthma were associated with insomnia disorder. These findings are in line with a survey that demonstrated that insomnia disorder was most strongly associated with psychiatric conditions, conditions characterised by psychological properties, and pain conditions [9]. Also, an association between gastroesophageal reflux disease and sleep problems has previously been reported [35].

In the present study a majority of subjects with insomnia disorder expressed a need for treatment, while $20.0 \%$ used prescribed sleep medication regularly. Other surveys from different countries report that a majority of subjects with insomnia do not use sleep medication. Rates of sleep medication usage in subjects with insomnia range from $21.5 \%$ to $33.2 \%[6,14]$. Variation in usage may be due to different definitions of sleep medication usage, but also cultural, social, or economic dissimilarities as well as differences in attitudes to sleep complaints and sleep medication.

\section{Conclusions}

This study provides important information about several aspects of the epidemiology of insomnia disorder, and the 
results have public health implications. The prevalence of insomnia disorder was $10.5 \%$, and it did not increase with advancing age. This may suggest that many elderly adapt their life stylein such a way that potential daytime consequences of disturbed sleep are not manifested. Insomnia disorder was strongly related to physical and psychiatric disorders, most notably depression, underlining the importance of investigating and treating insomnia in subjects with physical and psychiatric disorders. The cause-effect relationship is difficult to establish, but there is evidence that treatment of insomnia disorder should be considered separately and independent of other cooccurring disorders [36]. A majority of subjects with insomnia disorder expressed a need for treatment, but only one-fifth used prescribed sleep medication. This study confirms that insomnia disorder is a public health issue, and improved recognition and adequate treatment strategies are required.

\section{Conflict of Interests}

The authors declare that there is no conflict of interests regarding the publication of this paper.

\section{Acknowledgments}

Research grants were provided by the Swedish Research Council, Stockholm Council Research Foundation, and SBU (the Swedish Council on Health Technology Assessment).

\section{References}

[1] M. M. Ohayon, "Epidemiology of insomnia: what we know and what we still need to learn," Sleep Medicine Reviews, vol. 6, no. 2, pp. 97-111, 2002.

[2] M. M. Ohayon and G. Bader, "Prevalence and correlates of insomnia in the Swedish population aged 19-75 years," Sleep Medicine, vol. 11, no. 10, pp. 980-986, 2010.

[3] American Psychiatric Association, Diagnostic and Statistical Manual of Mental Disorders (DSM-IV-R), American Psychiatric Association, Washington, DC, USA, 4th edition, 2000.

[4] World Health Organisation, International Statistical Classification of Diseases and Related Health Problems (ICD), 10th revision, World Health Organisation, Geneva, Switzerland, 2nd edition, 1994.

[5] American Academy of Sleep Medicine, International Classification of Sleep Disorders: Diagnostic and Coding Manual (ICSD), American Sleep Disorders Association, Rochester, Minn, USA, 2nd edition, 2005.

[6] M. M. Ohayon and T. Sagales, "Prevalence of insomnia and sleep characteristics in the general population of Spain," Sleep Medicine, vol. 11, no. 10, pp. 1010-1018, 2010.

[7] S. Pallesen, I. H. Nordhus, G. H. Nielsen et al., "Prevalence of insomnia in the adult Norwegian population," Sleep, vol. 24, no. 7, pp. 771-779, 2001.

[8] B. Zhang and Y. K. Wing, "Sex differences in insomnia: a metaanalysis," Sleep, vol. 29, no. 1, pp. 85-93, 2006.

[9] B. Sivertsen, S. Krokstad, S. Øverland, and A. Mykletun, "The epidemiology of insomnia: associations with physical and mental health. The HUNT-2 study," Journal of Psychosomatic Research, vol. 67, no. 2, pp. 109-116, 2009.
[10] M. M. Ohayon and C. F. Reynolds III, "Epidemiological and clinical relevance of insomnia diagnosis algorithms according to the DSM-IV and the International Classification of Sleep Disorders (ICSD)," Sleep Medicine, vol. 10, no. 9, pp. 952-960, 2009.

[11] D. J. Taylor, L. Mallory, K. L. Lichstein, H. H. Durrence, B. W. Riedel, and A. J. Bush, "Comorbidity of chronic insomnia with medical problems," Sleep, vol. 30, pp. 213-218, 2007.

[12] R. Stewart, A. Besset, P. Bebbington et al., "Insomnia comorbidity and impact and hypnotic use by age group in a national survey population aged 16 to 74 years," Sleep, vol. 29, no. 11, pp. 1391-1397, 2006.

[13] M. M. Ohayon, M. Caulet, and P. Lemoine, "Comorbidity of mental and insomnia disorders in the general population," Comprehensive Psychiatry, vol. 39, no. 4, pp. 185-197, 1998.

[14] C. M. Morin, M. LeBlanc, M. Daley, J. P. Gregoire, and C. Mérette, "Epidemiology of insomnia: prevalence, self-help treatments, consultations, and determinants of help-seeking behaviors," Sleep Medicine, vol. 7, no. 2, pp. 123-130, 2006.

[15] P. Rhode, P. M. Lewinsohn, and J. R. Seeley, "Comparability of telephone and face to face interviews in assessing axis I and axis II disorders," The American Journal of Psychiatry, vol. 154, no. 11, pp. 1593-1598, 1997.

[16] M. M. Ohayon, "Nocturnal awakenings and difficulty resuming sleep: their burden in the European general population," Journal of Psychosomatic Research, vol. 69, no. 6, pp. 565-571, 2010.

[17] C. Nord, A. Mykletun, and S. D. Fosså, "Cancer patients' awareness about their diagnosis: a population-based study," Journal of Public Health Medicine, vol. 25, no. 4, pp. 313-317, 2003.

[18] L. Mallon and J. Hetta, "Detecting depression in questionnaire studies: comparison of a single question and interview data in community sample of older adults," European Journal of Psychiatry, vol. 16, no. 3, pp. 135-144, 2002.

[19] L. Mallon, J. E. Broman, and J. Hetta, "Relationship between insomnia, depression, and mortality: a 12-year follow-up of older adults in the community," International Psychogeriatrics, vol. 12, no. 3, pp. 295-306, 2000.

[20] L. Mallon, J. E. Broman, and J. Hetta, "Sleep complaints predict coronary artery disease mortality in males: a 12-year follow-up study of a middle-aged Swedish population," Journal of Internal Medicine, vol. 251, no. 3, pp. 207-216, 2002.

[21] L. Mallon, J. E. Broman, and J. Hetta, "High incidence ofdiabetes in men with sleep complaints or short sleep duration: a 12-year follow-up study of a middle-aged population," Diabetes Care, vol. 28, no. 11, pp. 2762-2767, 2005.

[22] E. O. Bixler, A. Kales, C. R. Soldatos, J. D. Kales, and S. Healey, "Prevalence of sleep disorders in the Los Angeles metropolitan ares," The American Journal of Psychiatry, vol. 136, no. 10, pp. 1257-1262, 1979.

[23] M. M. Ohayon and M. Partinen, "Insomnia and global sleep dissatisfaction in Finland," Journal of Sleep Research, vol. 11, no. 4, pp. 339-346, 2002.

[24] M. A. Grandner, J. L. Martin, N. P. Patel et al., "Age and sleep disturbances among American men and women: data from the U.S. Behavioral Risk Factor Surveillance System," Sleep, vol. 35, no. 3, pp. 395-406, 2012.

[25] A. J. Barsky, H. M. Peekna, and J. F. Borus, "Somatic symptom reporting in women and men," Journal of General Internal Medicine, vol. 16, no. 4, pp. 266-275, 2001.

[26] S. Nolen-Hoeksema, "Sex differences in unipolar depression: evidence and theory," Psychological Bulletin, vol. 101, no. 2, pp. 259-282, 1987. 
[27] E. McGrath, G. P. Keita, B. R. Strickland, and N. F. Russo, "Women and depression: risk factors and treatment issues," Final Report, The American psychological Association's National Task Force on Women and Depression, 1990.

[28] L. Lampio, P. Polo-Kantola, O. Polo, T. Kauko, J. Aittokallio, and T. Saaresranta, "Sleep in midlife women: effects of menopause, vasomotor symptoms, and depressive symptoms," Menopause. In press.

[29] D. L. Bliwise, "Sleep in normal aging and dementia," Sleep, vol. 16, no. 1, pp. 40-81, 1993.

[30] D. J. Dijk, J. A. Groeger, N. Stanley, and S. Deacon, "Age-related reduction in daytime sleep propensity and nocturnal slow wave sleep," Sleep, vol. 33, no. 2, pp. 211-223, 2010.

[31] E. B. Klerman and D. J. Dijk, "Age-Related Reduction in the Maximal Capacity for Sleep-Implications for Insomnia," Current Biology, vol. 18, no. 15, pp. 1118-1123, 2008.

[32] D. J. Dijk, J. F. Duffy, E. Kiel, T. L. Shanahan, and C. A. Czeisler, "Ageing and the circadian and homeostatic regulation of human sleep during forced desynchrony of rest, melatonin and temperature rhythms," Journal of Physiology, vol. 516, no. 2, pp. 611-627, 1999.

[33] M. M. Ohayon, J. Zulley, C. Guilleminault, S. Smirne, and R. G. Priest, "How age and daytime activities are related to insomnia in the general population: consequences for older people," Journal of the American Geriatrics Society, vol. 49, no. 4, pp. 360-366, 2001.

[34] M. M. Ohayon and S. C. Hong, "Prevalence of insomnia and associated factors in South Korea," Journal of Psychosomatic Research, vol. 53, no. 1, pp. 593-600, 2002.

[35] C. Jansson, H. Nordenstedt, M. A. Wallander et al., "A population-based study showing an association between gastroesophageal reflux disease and sleep problems," Clinical Gastroenterology and Hepatology, vol. 7, no. 9, pp. 960-965, 2009.

[36] L. Culpepper, "Secondary insomnia in the primary care setting: review of diagnosis, treatment, and management," Current Medical Research and Opinion, vol. 22, no. 7, pp. 1257-1268, 2006. 


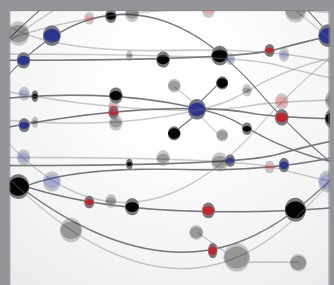

The Scientific World Journal
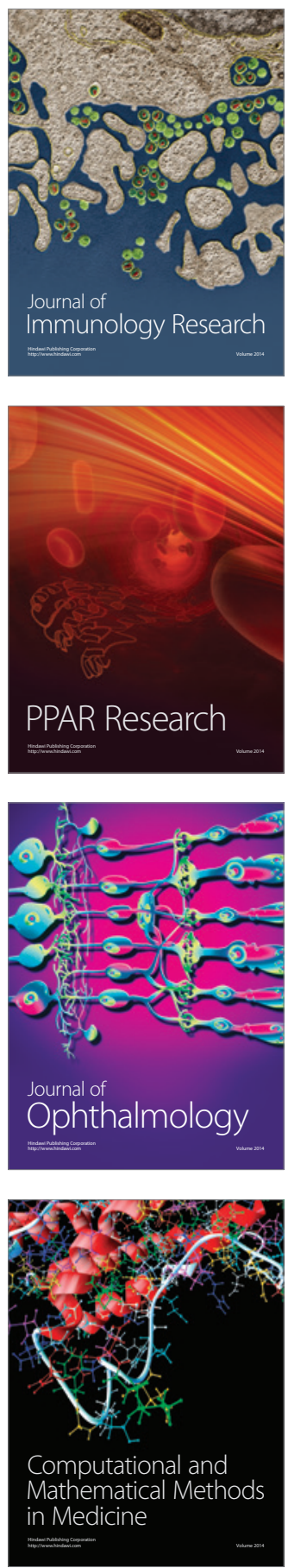

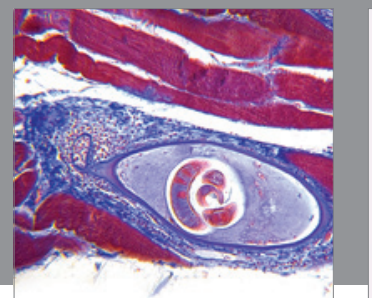

Gastroenterology

Research and Practice
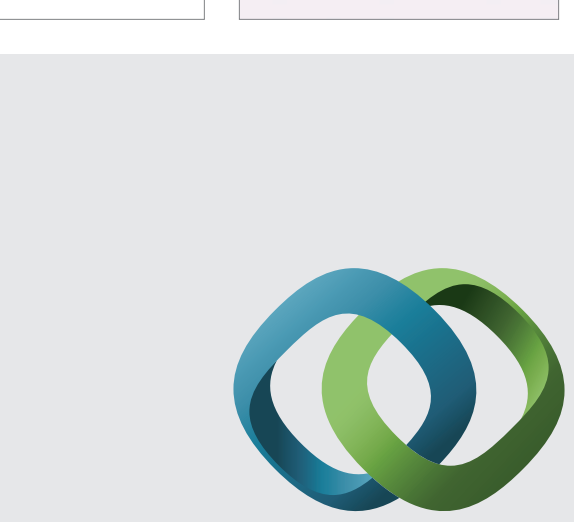

\section{Hindawi}

Submit your manuscripts at

http://www.hindawi.com
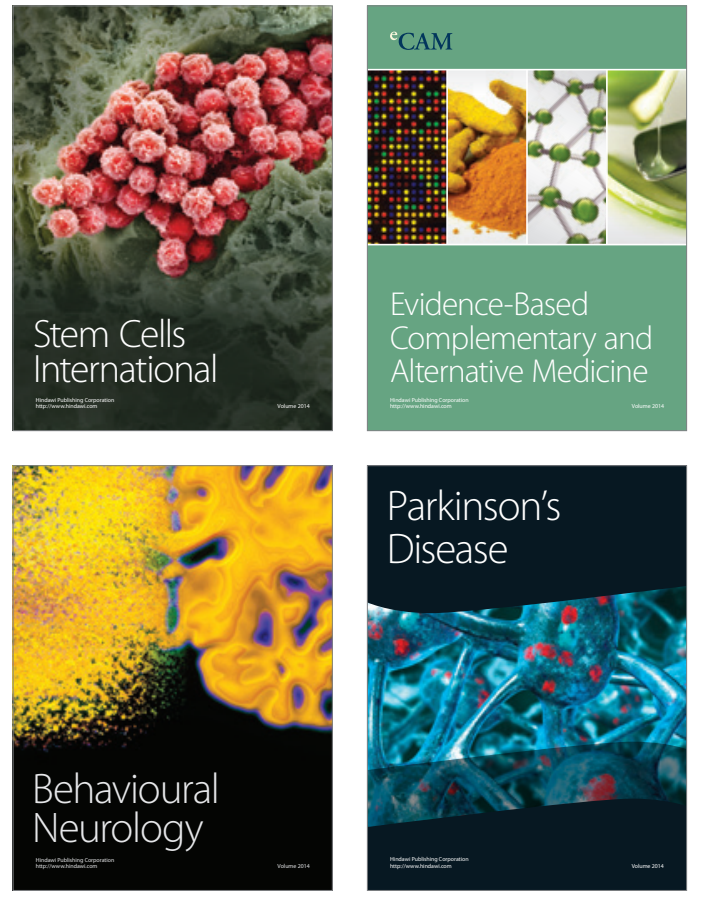
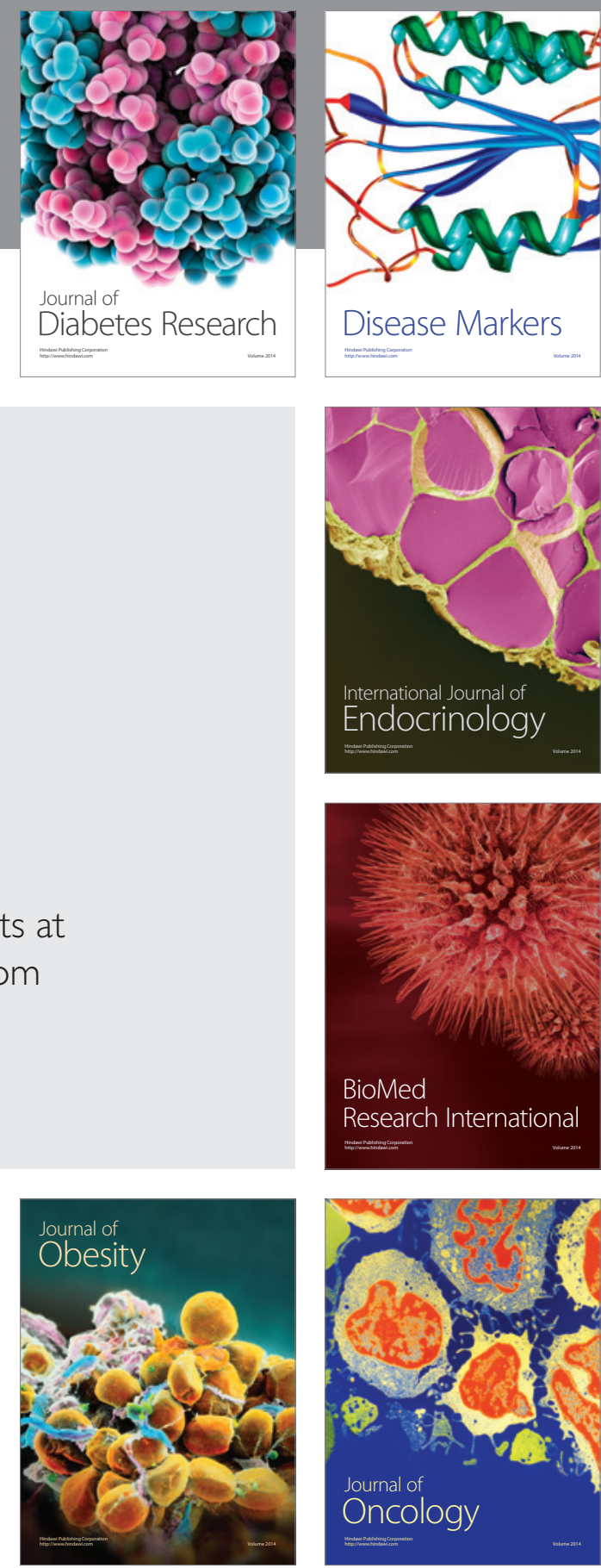

Disease Markers
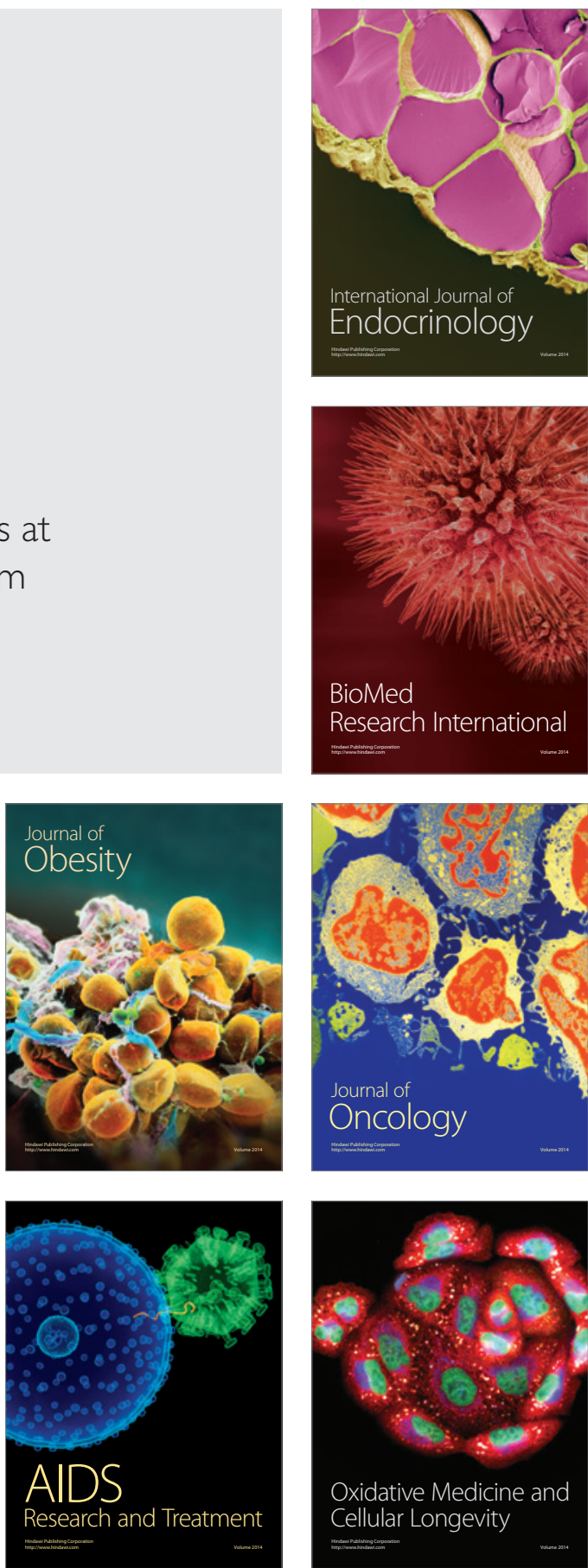